\title{
Universality of weak localization in disordered wires
}

\author{
C. W. J. Beenakker \\ Instituut-Lorentz, University of Leiden, P.O. Box 9506, 2300 RA Leiden, The Netherlands
}

(Received 20 August 1993)

\begin{abstract}
We compute the quantum correction $\delta A$ due to weak localization for transport properties $A=\sum_{n} a\left(T_{n}\right)$ of disordered quasi-one-dimensional conductors, by integrating the DorokhovMello-Pereyra-Kumar equation for the distribution of the transmission eigenvalues $T_{n}$. The result $\delta A=(1-2 / \beta)\left[\frac{1}{4} a(1)+\int_{0}^{\infty} d x\left(4 x^{2}+\pi^{2}\right)^{-1} a\left(\cosh ^{-2} x\right)\right]$ is independent of sample length or mean free path, and has a universal $1-2 / \beta$ dependence on the symmetry index $\beta \in\{1,2,4\}$ of the ensemble of scattering matrices. This result generalizes the theory of weak localization for the conductance to all linear statistics on the transmission eigenvalues.
\end{abstract}

Weak localization is a quantum transport effect which manifests itself as a magnetic-field-dependent correction to the classical Drude conductance. Discovered in $1979,{ }^{1,2}$ it was the first known quantum interference effect on a transport property. (For reviews, see Ref. 3.) At zero temperature, and in the quasi-one-dimensional (quasi-1D) limit $L \gg W$ of a long and narrow wire (length $L$, width $W$ ), the weak-localization correction to the conductance takes the universal form ${ }^{4,5}$

$$
\delta G=\frac{2 e^{2}}{h} \times\left\{\begin{array}{cl}
-\frac{1}{3} & (\beta=1) \\
0 & (\beta=2) \\
\frac{1}{6} & (\beta=4)
\end{array}\right.
$$

depending on the symmetry index $\beta$ of the ensemble of scattering matrices, but independent of microscopic parameters as sample length $L$ or mean free path $l$. If timereversal symmetry is broken (e.g., by a sufficiently strong magnetic field), then $\beta=2$ and $\delta G=0$. In the presence of both time-reversal and spin-rotation symmetry, $\beta=1$ and $\delta G<0$. If only the latter symmetry is broken (i.e., for strong spin-orbit scattering in zero magnetic field), then $\beta=4$ and $\delta G>0$. The implication for the magnetoconductance is that $G$ shows either a peak or a dip around zero field, depending on the presence or absence of strong spin-orbit scattering. The peak is precisely half as large as the dip.

The purpose of this paper is to demonstrate that the universality of the weak-localization correction expressed by Eq. (1) is generic for a whole class of transport properties, of which the conductance is but a special example. We consider a general transport property $A$ of the form

$$
A=\sum_{n=1}^{N} a\left(T_{n}\right)
$$

This is the definition of a linear statistic on the transmission eigenvalues $T_{1}, T_{2}, \ldots, T_{N}$. The word "linear" indicates that $A$ does not contain products of different $T_{n}$ 's, but the function $a(T)$ may well depend nonlinearly on $T$. The conductance is a special case for which $a(T)=\left(2 e^{2} / h\right) T$ is linear in $T$ (Landauer's formula). Other examples of linear statistics include the shot-noise power [with $a(T)$ a quadratic function], the conductance of a normal-superconductor interface [with $a(T)$ a rational function], and the supercurrent through a pointcontact Josephson junction [with $a(T)$ an algebraic function]. In Ref. 6 it was shown that the theory of "universal conductance fluctuations" can be generalized to all these linear statistics. Here we wish to establish such generality for the theory of weak localization. ${ }^{7}$

Our final result is a formula

$\delta A=\left(1-\frac{2}{\beta}\right)\left(\frac{1}{4} a(1)+\int_{0}^{\infty} d x \frac{a\left(\cosh ^{-2} x\right)}{4 x^{2}+\pi^{2}}\right)$

for the weak-localization correction $\delta A$ to the ensemble average $\langle A\rangle=A_{0}+\delta A$ of an arbitrary linear statistic $A$ of the form (2). The term $\delta A$ is a quantum correction of order $N^{0}$ to the classical $\beta$-independent value $A_{0}$, which is of order $N$ (with $N \gg 1$ being the number of scattering channels in the conductor). One easily verifies that substitution of $a(T)=\left(2 e^{2} / h\right) T$ into Eq. (3) yields the known result (1), using

$$
\int_{0}^{\infty} d x\left(4 x^{2}+\pi^{2}\right)^{-1}(\cosh x)^{-2}=\frac{1}{12}
$$

The fundamental significance of Eq. (3) is that it demonstrates that all linear statistics have a weak-localization correction which (i) is independent of sample length or mean free path, and (ii) has a $1-2 / \beta$ dependence on the symmetry index. In addition, Eq. (3) reduces the computation of the numerical value of the quantum correction to a quadrature, regardless of the complexity of the function $a(T) .^{8}$

The starting point of the analysis is the DorokhovMello-Pereyra-Kumar equation ${ }^{9}$

$$
\frac{\partial P}{\partial s}=\frac{2}{\beta N+2-\beta} \sum_{i=1}^{N} \frac{\partial}{\partial \lambda_{i}} \lambda_{i}\left(1+\lambda_{i}\right) J \frac{\partial}{\partial \lambda_{i}} J^{-1} P
$$

for the evolution of an ensemble of quasi-1D conductors of increasing length. For each ratio $s=L / l$ the ensemble is characterized by the probability distribution $P\left(\left\{\lambda_{n}\right\}, s\right)$ of the set of variables $\left\{\lambda_{n}\right\}=\lambda_{1}, \lambda_{2}, \ldots, \lambda_{N}$. The $\lambda$ variables are defined by $\lambda_{n}=\left(1-T_{n}\right) / T_{n}$ in terms of the transmission eigenvalues $T_{n}$. Since $T_{n} \in[0,1], \lambda_{n} \in$ 
$[0, \infty)$. The $N$ degrees of freedom in Eq. (4) are coupled by the factor $J\left(\left\{\lambda_{n}\right\}\right)=\prod_{2<\jmath}\left|\lambda_{2}-\lambda_{\jmath}\right|^{\beta}$, which is the Jacobian from the space of scattering matrices to the space of transmission eigenvalues. ${ }^{10}$ An exact solution of Eq. (4) is known, ${ }^{11}$ but only for the case $\beta=2$. This is of no use here, since weak localization is absent for $\beta=2$. We therefore employ a different method, which yields for any $\beta$ the eigenvalue density in the large- $N$ limit. (This is the relevant limit for weak localization, which requires $l \ll L \ll N l$.) The key technical ingredient is an asymptotic expansion published by Dyson more than 20 years ago, ${ }^{12}$ but which had remained largely unnoticed.

We seek to reduce Eq. (4) to an equation for the density $\rho(\lambda, s)=\left\langle\sum_{n} \delta\left(\lambda-\lambda_{n}\right)\right\rangle$ of the $\lambda$-variables. The brackets \langle\rangle denote an average over $\left\{\lambda_{n}\right\}$ with distribution $P\left(\left\{\lambda_{n}\right\}, s\right)$. Multiplying both sides of Eq. (4) by $\sum_{n} \delta\left(\lambda-\lambda_{n}\right)$ and integrating over $\lambda_{1}, \lambda_{2}, \ldots, \lambda_{N}$ one obtains an equation

$$
\begin{aligned}
& \frac{\partial \rho}{\partial s}=\frac{2}{\beta N+2-\beta} \frac{\partial}{\partial \lambda} \lambda(1+\lambda)\left(\frac{\partial \rho}{\partial \lambda}-\beta I\right), \\
& I(\lambda, s)=\int_{0}^{\infty} d \lambda^{\prime} \rho_{2}\left(\lambda, \lambda^{\prime}, s\right)\left(\lambda-\lambda^{\prime}\right)^{-1},
\end{aligned}
$$

which contains an integral over the pair distribution function

$$
\rho_{2}\left(\lambda, \lambda^{\prime}, s\right)=\left\langle\sum_{\imath \neq \jmath} \delta\left(\lambda-\lambda_{\imath}\right) \delta\left(\lambda^{\prime}-\lambda_{\jmath}\right)\right\rangle .
$$

To close Eq. (5) we use Dyson's asymptotic expansion ${ }^{12}$ $\frac{I(\lambda, s)}{\rho(\lambda, s)}=\int_{0}^{\infty} d \lambda^{\prime} \frac{\rho\left(\lambda^{\prime}, s\right)}{\lambda-\lambda^{\prime}}+\frac{1}{2} \frac{\partial}{\partial \lambda} \ln \rho(\lambda, s)+\mathcal{O}\left(N^{-1}\right)$.

Substitution into Eq. (5a) gives

$$
\begin{aligned}
\frac{\partial \rho}{\partial s}= & \frac{2}{\beta N+2-\beta} \frac{\partial}{\partial \lambda} \lambda(1+\lambda) \rho \frac{\partial}{\partial \lambda}\left(\left(1-\frac{1}{2} \beta\right) \ln \rho\right. \\
& \left.-\beta \int_{0}^{\infty} d \lambda^{\prime} \rho\left(\lambda^{\prime}, s\right) \ln \left|\lambda-\lambda^{\prime}\right|\right) .
\end{aligned}
$$

At this point it is convenient to switch to a new set of independent variables $\left\{x_{n}\right\}$, defined by $\lambda_{n}=\sinh ^{2} x_{n}$. Since $T_{n}=\left(1+\lambda_{n}\right)^{-1}$, one has $T_{n}=1 / \cosh ^{2} x_{n}$, with $x_{n} \in[0, \infty)$. The ratio $L / x_{n}$ has the physical interpretation of a channel-dependent localization length. ${ }^{10}$ The density $\tilde{\rho}(x, s)$ of the $x$-variables is related to $\rho(\lambda, s)$ by $\tilde{\rho}=\rho d \lambda / d x=\rho \sinh 2 x$. In terms of the new variables, Eq. (8) takes the form

$$
\begin{aligned}
\frac{\partial \tilde{\rho}}{\partial s}= & \frac{1}{2(N-\gamma)} \frac{\partial}{\partial x} \tilde{\rho} \frac{\partial}{\partial x}\left(\int_{0}^{\infty} d x^{\prime} \tilde{\rho}\left(x^{\prime}, s\right) u\left(x, x^{\prime}\right)\right. \\
& \left.-\frac{1}{2} \gamma V(x)-\frac{1}{2} \gamma \ln \tilde{\rho}(x, s)\right),
\end{aligned}
$$

with the definitions $\gamma=1-2 / \beta, V(x)=-\ln |\sinh 2 x|$, $u\left(x, x^{\prime}\right)=-\ln \left|\sinh ^{2} x-\sinh ^{2} x^{\prime}\right|$. We need to solve Eq. (9) to the same order in $N$ as the expansion (7), i.e., neglecting terms of order $N^{-1}$. To this end we decompose $\tilde{\rho}=\tilde{\rho}_{0}+\delta \tilde{\rho}$, with $\tilde{\rho}_{0}$ of order $N$ and $\delta \tilde{\rho}$ of order $N^{0}$. Substitution into Eq. (9) yields to order $N$ an equation for $\tilde{\rho}_{0}$,

$$
\frac{\partial \tilde{\rho}_{0}}{\partial s}=\frac{1}{2 N} \frac{\partial}{\partial x} \tilde{\rho}_{0} \frac{\partial}{\partial x} \int_{0}^{\infty} d x^{\prime} \tilde{\rho}_{0}\left(x^{\prime}, s\right) u\left(x, x^{\prime}\right) .
$$

This is essentially the problem solved by Mello and Pichard, ${ }^{13}$ who showed that

$$
\tilde{\rho}_{0}(x, s)=N s^{-1} \theta(s-x),
$$

in the relevant regime $s \gg 1, s \gg x$. [The function $\theta(\xi)$ equals 1 for $\xi>0$ and 0 for $\xi<0$.] Equation (11) implies that, to order $N$, the $x$-variables have a uniform density of $N l / L$, with a cutoff at $L / l$ such that $\int_{0}^{\infty} d x \tilde{\rho}_{0}=N$. In the cutoff region $x \sim L / l$ the density deviates from uniformity, but this region is irrelevant since the transmission eigenvalues are exponentially small for $x \gg 1$. One can readily verify by substitution that the solution (11) satisfies Eq. (10), using

$$
\frac{\partial}{\partial x} \int_{0}^{s} d x^{\prime} u\left(x, x^{\prime}\right)=-2 x \text { for } s \gg 1, s \gg x .
$$

Now we are ready to compute the $\mathcal{O}\left(N^{0}\right)$ correction $\delta \tilde{\rho}$ to the density. Substituting $\tilde{\rho}=\tilde{\rho}_{0}+\delta \tilde{\rho}$ into Eq. (9), and using Eqs. (11) and (12), we find

$$
\begin{array}{r}
\frac{\partial \delta \tilde{\rho}}{\partial s}=\frac{1}{2 s} \frac{\partial^{2}}{\partial x^{2}} \int_{0}^{\infty} d x^{\prime} \delta \tilde{\rho}\left(x^{\prime}, s\right) u\left(x, x^{\prime}\right) \\
-\frac{1}{s} \frac{\partial}{\partial x}(x \delta \tilde{\rho})-\frac{\gamma}{4 s} \frac{\partial^{2} V}{\partial x^{2}}-\frac{\gamma}{s^{2}} .
\end{array}
$$

The last term $\gamma / s^{2}$ on the right-hand side is a factor $s$ smaller than the other terms, and may be neglected for $s \gg 1$. Equation (13) thus has the $s$-independent solution $\delta \tilde{\rho}(x)$ satisfying

$$
\begin{aligned}
\frac{1}{2} \frac{d^{2}}{d x^{2}} \int_{0}^{\infty} d x^{\prime} \delta \tilde{\rho}\left(x^{\prime}\right) \ln \left|\sinh ^{2} x-\sinh ^{2} x^{\prime}\right| \\
+\frac{d}{d x}[x \delta \tilde{\rho}(x)]=\frac{\gamma}{4} \frac{d^{2}}{d x^{2}} \ln |\sinh 2 x|
\end{aligned}
$$

It remains to solve the integrodifferential equation (14). This can be done analytically by means of the identity $^{14}$

$$
\begin{aligned}
\int_{0}^{\infty} d x^{\prime} f\left(x^{\prime}\right) \ln \mid & \sinh ^{2} x-\sinh ^{2} x^{\prime} \mid \\
& =\int_{-\infty}^{\infty} d x^{\prime} f\left(\left|x^{\prime}\right|\right) \ln \left|\sinh \left(x-x^{\prime}\right)\right|
\end{aligned}
$$

which transforms the integration into a convolution. The Fourier transform then satisfies an ordinary differential equation, which is easily solved. The result is

$$
\delta \tilde{\rho}(x)=(1-2 / \beta)\left[\frac{1}{2} \delta(x)+\left(4 x^{2}+\pi^{2}\right)^{-1}\right],
$$

as one can also verify directly by substitution into Eq. (14). The correction (16) to the uniform density (11) takes the form of a deficit (for $\beta=1$ ) or an excess (for $\beta=4$ ), concentrated in the region $x \lesssim 1$. For $\beta=2$ 
there is no $\mathcal{O}\left(N^{0}\right)$ deviation from uniformity. The existence of a $\beta$-dependent density excess or deficit in the metallic regime was anticipated by Stone. Mello, Muttalib. and Pichard ${ }^{10}$ from the $\beta$-dependence of the localization length in the insulating regime. However. as emphasized by these authors. their argument is simply suggestive and needs to be made quantitative. Equation (16) does that.

The weak-localization correction $\delta A$ follows upon integration,

$$
\delta .4=\int_{0}^{\infty} d x \delta \tilde{\rho}(x) a\left(1 / \cosh ^{2} x\right) .
$$

Combination of Eqs. (16) and (17) finally gives the formula (3) for the weak-localization correction to the ensemble average of an arbitrary linear statistic, as advertized in the introduction.

We conclude with an illustrative application of Eq. (3). to the conductance $G_{\mathrm{NS}}$ of a disordered normal-metalsuperconductor (NS) junction. This transport property is a linear statistic for zero magnetic field. ${ }^{15}$

$$
G_{\mathrm{NS}}=\frac{2 e^{2}}{h} \sum_{n=1}^{N} \frac{2 T_{n}^{2}}{\left(2-T_{n}\right)^{2}}
$$

In a semiclassical treatment, the ensemble average $\left\langle G_{\mathrm{NS}}\right\rangle$ is just the Drude conductance - unaffected by Andreev reflection at the NS interface. However. the quantum correction $\delta G_{\text {NS }}$ due to weak localization is enhanced by Andreev reflection. ${ }^{15}$ Previously. there was no method to calculate $\delta G_{\mathrm{NS}}{ }^{8}$ Now. using Eq. (3) one computes (for $\beta=1) \delta G_{\mathrm{NS}}=-\left(1-4 \pi^{-2}\right)\left(2 e^{2} / h\right)$. which exceeds the result $\delta G=-\frac{1}{3}\left(2 e^{2} / h\right)$ in the normal state by almost a factor of 2. The experimental observation of the enhancement of weak localization by Andreev reflection has recently been reported. ${ }^{16}$

In summary, we have shown that the universality of the weak-localization effect in disordered wires is generic for a whole class of transport properties. viz.. the class of linear statistics on the transmission eigenvalues. A formula has been derived which permits the computation of the weak-localization correction in cases that previous methods were not effective. This quantum correction is independent of sample length or mean free path. and has a $1-2 / \beta$ dependence on the symmetry index. for all linear statistics.

Discussions with B. Rejaei and F. L. J. Vos are gratefully acknowledged. This research was supported in part by the Dutch Science Foundation NWO/FOM.
${ }^{1}$ P. W. Anderson, E. Abrahams, and T. V. Ramakrishnan, Phys. Rev. Lett. 43, 718 (1979).

${ }^{2}$ L. P. Gor'kov, A. I. Larkin, and D. E. Khmel'nitskiř, Pis'ma Zh. Eksp. Teor. Fiz. 30, 248 (1979) [JETP Lett. 30, 228 (1979)].

${ }^{3}$ G. Bergmann, Phys. Rep. 107, 1 (1984); P. A. Lee and T. V. Ramakrishnan, Rev. Mod. Phys. 57, 287 (1985); S. Chakravarty and A. Schmid, Phys. Rep. 140, 193 (1986).

${ }^{4}$ P. A. Mello and A. D. Stone, Phys. Rev. B 44, 3559 (1991).

${ }^{5}$ The wire geometry $L \gg W$ is essential for the universality of Eq. (1): in a square or cube geometry $\delta G$ acquires a dependence on $L$ and $l$, cf. Ref. 3 . The zero-temperature limit is also essential: $\delta G$ becomes dependent on the phasecoherence length $l_{\phi}$ if $l_{\phi}<L$.

${ }^{6}$ C. W. J. Beenakker, Phys. Rev. Lett. 70, 1155 (1993); Phys. Rev. B 47, 15763 (1993).

${ }^{7}$ Although Ref. 6 also deals with a general theory of quantum transport for linear statistics, both the starting point and the goal are different. Reference 6 addresses the fluctuations around the ensemble average, by means of a randommatrix theory which by construction contains no information on the ensemble average. The present paper, in contrast, addresses the ensemble average itself, which is where the weak-localization effect plays a role.

${ }^{8}$ Previous theoretical work on weak localization has either been based on diagrammatic perturbation theory, (Refs. 1-3), or on a moment expansion of Eq. (4) (Ref. 4). Both methods are unsuitable for arbitrary $a(T)$, and thus can- not be used for the present purpose. In fact, apart from the conductance, the only other quantity for which the weak-localization correction has been calculated previously is the shot-noise power; see M. J. M. de Jong and C. W. J. Beenakker, Phys. Rev. B 46, 13400 (1992).

${ }^{9}$ P. A. Mello, P. Pereyra, and N. Kumar, Ann. Phys. 181, 290 (1988); O. N. Dorokhov, Pis'ma Zh. Eksp. Teor. Fiz. 36, 259 (1982) [JETP Lett. 36, 318 (1982)].

${ }^{10}$ A. D. Stone, P. A. Mello, K. A. Muttalib, and J.-L. Pichard, in Mesoscopıc Phenomena in Solrds, edited by B. L. Al'tshuler, P. A. Lee, and R. A. Webb (North-Holland, Amsterdam, 1991).

${ }^{11}$ C. W. J. Beenakker and B. Rejaei, Phys. Rev. Lett. (to be published)

${ }^{12}$ F. J. Dyson, J. Math. Phys. 13, 90 (1972). The asymptotic expansion (7) was derived by Dyson for a problem where $\lambda$ is free to vary from $-\infty$ to $\infty$. In Ref. 6 it is shown that the restriction $\lambda \geq 0$ introduces no extra terms, to the order considered.

${ }^{13}$ P. A. Mello and J.-L. Pichard. Phys. Rev. B 40. 5276 (1989).

${ }^{14} \mathrm{I}$ am indebted to B. Rejaei for teaching me this method of solution.

${ }^{15}$ C. W. J. Beenakker, Phys. Rev. B 46, 12841 (1992): I. K. Marmorkos, C. W. J. Beenakker. and R. A. Jalabert, $\imath b \imath d$. 48, 2811 (1993).

${ }^{16}$ K.-M. H. Lenssen et al, Surf. Sci. (to be published). 